\title{
EL FUTURO DE LA “FLOR EUROPEA": LA NUEVA ETIQUETA ECOLÓGICA DE LA UE
}

\author{
BLANCA LOZANO CUTANDA \\ Catedrática de Derecho Administrativo \\ Universidad del País Vasco / Euskal Herriko Unibertsitatea \\ Consejera Académica \\ Gómez-Acebo \& Pombo \\ blanca.lozano@ehu.es
}

Recibido: 3 de abril 2010 / Aceptado: 27 de mayo de 2010

RESUMEN: La ecoetiqueta de la Unión Europea es un sistema voluntario de calificación ambiental que se introdujo en 1992. Sin embargo, resulta todavía desconocido para una gran mayoría de consumidores europeos. Por ello, se ha llevado a cabo un proceso de revisión del sistema que culmina con el nuevo Reglamento (CE) $\mathrm{n}^{\mathrm{o}}$ $66 / 2010$, cuyo contenido es analizado en la presente nota.

RESUM: L'ecoetiqueta de la Unió Europea és un sistema voluntari de qualificació ambiental introduït el 1992. Tanmateix, resulta encara desconegut per a la gran majoria dels consumidors europeus. Per aquest motiu, s'ha dut a terme un procés de revisió del sistema que culmina amb el nou Reglament (CE) núm. 66/2010, el contingut del qual és analitzat en aquesta nota.

\begin{abstract}
The EU ecolabel is a voluntary system of environmental qualification which was introduced in 1992. However, it is still unknown for the great majority of European consumers. For this reason it has been carried out a process of revision of the
\end{abstract}


system which culminates in the new Regulation (EC) No 66/2010, whose contents are analyzed in this note.

PALABRAS CLAVE: Ecoetiqueta - Unión Europea - Consumidores

PARAULES CLAU: Ecoetiqueta — Unió Europea - Consumidors

KEYWORDS: Ecolabel — European Union - Consumers

Sumario: I. Introducción. II. Ámbito de aplicación del sistema. III. Procedimiento y autoridades competentes para otorgar la etiqueta ecológica de la UE. 1. La elaboración y establecimiento de los criterios de la etiqueta ecológica. 2. La verificación de los productos y la concesión de la ecoetiqueta. IV. Medidas para promover la etiqueta ecológica de la UE

\section{INTRODUCCIÓN}

Los sistemas de etiquetado ecológico, ya sean públicos o puramente privados, constituyen un mecanismo de incentivo para la mejora del comportamiento ambiental de empresas y otras organizaciones. Se trata de arbitrar un procedimiento para conceder, a aquellos productos, servicios o empresas que cumplan determinados requisitos 
ecológicos, una marca o logotipo de conformidad ecológica que acredite la "calidad verde" del producto, del servicio, o de la empresa en cuestión.

Las ecoetiquetas pueden otorgarse a las empresas para certificar su participación en un sistema de ecogestión (como ocurre en el caso de la ISO 14-001, o en el Sistema de ecogestión y ecoauditoría europeo EMAS, "eco-management and audit scheme" ), o bien concederse directamente a los productos o servicios que demuestren que cumplen determinados requisitos o ventajas ambientales que les acreditan como respetuosos del medio ambiente.

A su vez, dada la multiplicidad de marcas ambientales que resultan aplicables a los productos o servicios, se han aprobado normas ISO que definen tres tipos de mecanismos diferentes:

1. las etiquetas tipo I, también conocidas como ecoetiquetas y a las que se adscribe la etiqueta ecológica de la Unión Europea, son sistemas voluntarios de calificación ambiental que identifican y certifican de forma oficial que ciertos productos o servicios tienen una menor afección sobre el medio ambiente (la norma ISO aplicable para este tipo de etiquetas es la ISO 14024:2005);

2. las autodeclaraciones ambientales o etiquetas del tipo II, que son realizadas por el propio fabricante en forma de textos, símbolos o gráficos y que no necesariamente son certificadas por una tercera parte independiente (los requerimientos específicos de este tipo de etiquetas se recogen en la norma ISO 1421:2005);

3. las declaraciones ambientales de productos (DAP) o etiquetas tipo III, son una nueva forma de declaración ambiental, que ofrece información sobre el impacto ambiental de un producto o servicio a través de análisis del ciclo de vida. Se ha desarrollado para evitar algunas de las dificultades que se han presentado con los esquemas de etiquetado Tipo I en la que se obliga a un que solamente un porcentaje bajo de productos de una categoría pudieran cumplir con los criterios ecológicos establecidos (están reguladas por la norma ISO 14025:2005).

\footnotetext{
${ }^{1}$ Regulado por el Reglamento (CE) no 761/2001, del Parlamento Europeo y del Consejo, de 19 de marzo de 2001, por el que se permite que las organizaciones se adhieran con carácter voluntario a un sistema comunitario de gestión y auditorías medioambientales (EMAS).
} 
El etiquetado ecológico Tipo I que aquí nos ocupa presenta las siguientes características: (i) es un etiquetado voluntario; (ii) supone el otorgamiento de una etiqueta cuando el producto cumple ciertos criterios; (iii) los criterios de aprobación/desaprobación son establecidos para cada categoría de producto después de considerar los impactos en todo su ciclo de vida del producto; (iv) los criterios están disponibles al público.

Dos son, fundamentalmente, los objetivos que se persiguen con la implantación del etiquetado ecológico por los organismos oficiales encargados de reconocer las marcas:

a) Incentivar las actividades productivas respetuosas con el medio ambiente mediante la recompensa que supone el otorgamiento de un logotipo ecológico que, en un mercado cada vez más sensibilizado por las cuestiones ambientales, permite mejorar la imagen del producto y de la empresa, y ejerce por ello un efecto positivo en las ventas. Se logra, de esta forma, que el marcado interés de la sociedad actual por la protección ambiental actúe como fuerza motriz de las decisiones productivas y comerciales de las empresas.

b) Ofrecer a los consumidores una información exacta y verídica sobre las repercusiones ecológicas de los productos o servicios que les permita orientar su compra hacia los menos perjudiciales para el medio ambiente. Se trata de que entre productos similares, cuya calidad está garantizada, se promueva aquél que ofrezca ventajas ecológicas adicionales.

Ha de tenerse en cuenta, en este sentido, que la publicidad "ecológica" engañosa o fraudulenta es un problema creciente en los países de nuestro entorno, al utilizarse cada vez más el reclamo ambiental en los mensajes publicitarios. Términos como "producto ecológico", "reciclable", "degradable" o "amigo del ozono", son de uso común en un mercado en el que "lo verde vende" y se utilizan con frecuencia de forma fraudulenta o simplemente engañosa (así, por ejemplo, si se promueve un producto como "biodegradable" cuando su descomposición tarda cientos de años, lo que no le cualifica especialmente desde la perspectiva ambiental).

Resulta por ello muy importante que se establezcan, por un lado medios para garantizar la exactitud de las pretensiones ambientales, persiguiéndose jurídicamente la publicidad ambiental engañosa (lo que constituye hoy una demanda creciente de ecologistas y 
consumidores), y que se provea por otro lado al consumidor con un instrumento de información objetivo y veraz, avalado por las instituciones públicas, sobre la “ecocompatibilidad" de los productos, función esta que cumplen las ecoetiquetas objeto de estudio.

El programa de etiquetado ecológico de productos alemán, introducido en 1978 y que utiliza el logotipo del "Ángel azul” empleado en el Programa de las Naciones Unidas para el medio ambiente, ha sido el precursor y modelo de todos los demás, seguido por Canadá (1988), Japón (1989), y los países nórdicos (1989). En Francia, se ha creado la marca "NF-Medioambiental", y en Estados Unidos existen multitud de etiquetas ecológicas, como la conocida "the Green Seal".

El éxito de estas ecoetiquetas nacionales llevó a la Comunidad Europea a regular una etiqueta ecológica con validez en todo el ámbito europeo, cuya introducción se realizó mediante un Reglamento de 1992, relativo a un sistema comunitario de concesión de etiqueta ecológica, posteriormente sustituido por un Reglamento de 2000, relativo a un sistema comunitario revisado de concesión de etiqueta ecológica, que acaba, a su vez, de ser sustituido por el Reglamento (CE) no 66/2010 del Parlamento Europeo y del Consejo, de 25 de noviembre de 2009, relativo a la etiqueta ecológica de la $\mathrm{UE}^{2}$.

La ecoetiqueta europea y su logotipo, consistente en una flor cuyo centro es la "E" de Europa (por lo que se la conoce también como la "Flor Europea"), resulta todavía desconocido para la una gran mayoría de consumidores europeos, lo que demuestra el escaso éxito que ha tenido hasta ahora este sistema para conseguir su doble objetivo, reconocido por sus Reglamentos reguladores, de promover productos respetuosos con el medio ambiente y de proporcionar a los consumidores información, exacta y no engañosa, sobre dichos productos.

Se acometió por ello hace tres años un proceso de revisión del sistema que dio lugar, tras un procedimiento de evaluación y consulta, a la presentación por la Comisión de una "Propuesta de Reglamento del Parlamento Europeo y del Consejo relativo a un sistema ecológica comunitaria" [COM (2008) 401 final], que finalmente ha cristalizado en el nuevo Reglamento (CE) $n^{\circ}$ 66/2010 objeto de este comentario.

\footnotetext{
${ }^{2}$ En España, por Real Decreto 598/94, de 8 de abril, se aprobaron las normas para la aplicación del Reglamento de 1992, cuyas disposiciones han de considerarse en vigor, mientras no sean sustituidas y en tanto no resulten incompatibles con los preceptos del nuevo Reglamento europeo.
} 
En la Propuesta de nuevo Reglamento que presentó la Comisión se señalaba que, según los resultados de la evaluación llevada a cabo, el objetivo del sistema sigue siendo válido y deseable desde la perspectiva estratégica de la UE, por cuanto la ecoetiqueta “ofrece a los consumidores de la Unión una certificación medioambiental fiable y puede proporcionar a las empresas la posibilidad de utilizar una etiqueta para su marketing a nivel europeo o mundial".

Las razones que postulan a favor de un establecimiento amplio y efectivo del sistema de etiqueta ecológica en todos los países de la UE son ciertamente muy poderosas, tanto desde la perspectiva ambiental como desde la económica.

Por un lado, la existencia de una etiqueta ecológica común, cuyo logotipo puede ser reconocido por más de 450 millones de consumidores, potencia el poder de incentivo ambiental de este instrumento, al incrementar su eficacia económica y el ámbito de difusión de la información sobre las repercusiones ecológicas de los productos.

Pero, además, la existencia de una ecoetiqueta común a todos los Estados miembros coadyuva a la uniformidad y homologación del mercado único como requisito necesario para garantizar la libre competencia. Debe señalarse, en este sentido, que los programas de etiquetado ecológico nacionales, aunque son voluntarios y no discriminan en principio según la región o país de origen del producto, pueden contener y contienen con frecuencia determinadas prescripciones que dificultan la concesión de la etiqueta a fabricantes extranjeros (así ocurre, por ejemplo, cuando a los productos solicitantes se les exige el cumplimiento de ciertas normas nacionales o el sometimiento a una actividad de verificación y control llevada a cabo por las autoridades del país).

Sin embargo, a pesar de la bondad de sus objetivos, la etiqueta europea no acaba de despegar. Los datos que aportaba la Propuesta de nuevo Reglamento de la Comisión demuestran que la implantación del sistema es todavía muy insuficiente, y ello después de más de quince años de funcionamiento. Se señala así que aunque el sistema ha crecido recientemente en cuanto a número de empresas titulares de licencia, solo se han establecido 26 categorías de productos y solo hay alrededor de 500 empresas que fabrican productos con etiqueta ecológica. El total de ventas de productos con etiqueta ecológica asciende a mil millones de euros anuales aproximadamente, lo que representa una parte muy pequeña del mercado potencial de la UE. 
La Comisión concluía que, de acuerdo con los resultados del proceso de revisión a que se ha sometido el sistema, este insuficiente desarrollo de la ecoetiqueta se debe al escaso conocimiento de la etiqueta y a la poca aceptación por la industria, como consecuencia del exceso de gestión y procesos burocráticos.

El nuevo Reglamento se dirige, por ello, como destaca en sus considerandos, a "aumentar su eficacia y racionalizar su funcionamiento". Para ello, la nueva regulación pretende reducir las cargas burocráticas e introducir procedimientos más rápidos para la aprobación y revisión de los criterios para su otorgamiento, con el objetivo de que se incremente en número de categorías de productos incluidas en el sistema hasta un número de entre 40 y 50 en $2015^{3}$. La simplificación empieza por la propia normativa, pues el Reglamento contiene prácticamente toda la regulación del sistema y ya no se remite, como hacía el anterior, a decisiones a adoptar por la Comisión para regular aspectos tales como los contratos de utilización de la etiqueta o los cánones a pagar por los usuarios.

Las dificultades de implantación de la ecoetiqueta no se derivan, sin embargo, únicamente de las cargas burocráticas o de la complejidad de la regulación. Uno de los factores que más han incidido, sin duda, en la insuficiente implantación de la etiqueta ecológica europea ha sido su coexistencia con multitud de sistemas de ecoetiquetados nacionales. En el primer Reglamento que reguló el sistema debió preverse la progresiva sustitución de los sistemas de etiquetado nacionales preexistentes por el sistema comunitario en aras de la necesaria armonización europea. El que no se hiciera así se explica por la fuerte resistencia de Alemania y de los países nórdicos a renunciar a sus propias marcas de etiquetado ecológico, que habían alcanzado ya un importante arraigo e implantación social. Por ello, como se verá, el nuevo Reglamento adopta nuevas medidas tendentes a frenar la creación de nuevas etiquetas Tipo I y a procurar una armonización entre la ecoetiqueta europea y las nacionales ya existentes.

3 Véase la información difundida por la Comisión, "New rules strengthening the EU Ecolabel", en la página web: www.ec.europa.eu/environment/ecolabel/news/archives/2010/march/ecolabel_events_01.pdf 


\section{II. ÁMBITO DE APLICACIÓN DEL SISTEMA}

En el programa europeo de etiquetado ecológico participan todos los Estados miembros del Espacio Económico Europeo, esto es, los países integrantes de la Comunidad Europea y Noruega, Islandia y Liechtenstein.

En cuanto al ámbito objetivo de aplicación de la ecoetiqueta, se considera como "producto" a efectos de su participación en el sistema, "todo bien o servicio suministrado para distribución, consumo o utilización en el mercado comunitario, ya sea mediante pago o de forma gratuita" (art. 2).

El nuevo Reglamento ha ampliado de forma importante la posibilidad de utilizar la etiqueta ecológica de la UE, destacando, a este respecto dos medidas:

- Suprime la exclusión del sistema de los productos alimenticios y las bebidas que establecía el anterior Reglamento. Para los alimentos y piensos ya existe, sin embargo, una regulación de la UE dirigida a certificar, mediante el uso de determinados términos y un logotipo específico, el carácter ecológico de aquéllos que cumplan determinados requisitos y normas de producción [Reglamento (CE) 834/2007 del Consejo, de 28 de junio de 2007, sobre producción y etiquetado de los productos ecológicos]. Por ello, el Reglamento dispone que, antes de que se aprueben criterios para otorgar la etiqueta a esta categoría de productos la Comisión habrá de realizar un estudio para determinar la viabilidad de establecer unos criterios que cubran su comportamiento ambiental durante todo su ciclo de vida, incluidos los de la pesca y la acuicultura, en el que se deberá considerar la opción de que únicamente puedan acogerse a la etiqueta ecológica de la UE los productos certificados como ecológicos por el citado Reglamento, con el fin de evitar confusiones a los consumidores.

- Suprime las condiciones que antes se requerían para que un producto pudiera participar en el sistema, relativas a su volumen de ventas y comercio en el mercado europeo y a la importancia de sus efectos ambientales.

Actualmente, del ámbito de aplicación del Reglamento 3únicamente se excluyen los medicamentos para uso humano o veterinario y cualquier tipo de productos sanitarios. 


\section{PROCEDIMIENTO Y AUTORIDADES COMPETENTES PARA OTORGAR LA ETIQUETA ECOLÓGICA DE LA UE}

El procedimiento para otorgar la etiqueta ecológica comunitaria consta de dos fases: 1) la elaboración y establecimiento de los criterios específicos para cada categoría de productos, que establecerán los requisitos medioambientales que debe cumplir un producto para llevar la etiqueta ecológica de la UE; 2) la verificación de los productos aspirantes a su obtención a fin de determinar si se ajustan o no a los criterios establecidos.

\section{La elaboración y establecimiento de los criterios de la etiqueta ecológica}

La primera fase se llevaba a cabo hasta ahora exclusivamente a nivel comunitario, pero el nuevo Reglamento ha otorgado protagonismo a los Estados miembros y a los propios sectores interesados, reconociéndoles competencia para adoptar la iniciativa y dirigir la elaboración de los criterios.

A la Comisión le asiste en esta fase del procedimiento un organismo dedicado en exclusiva y de modo permanente al sistema comunitario de etiquetado ecológico, que se encarga de la elaboración y revisión de los criterios para la concesión de la ecoetiqueta, denominado Comité de Etiqueta Ecológica de la Unión Europea (CEEUE). De acuerdo con el nuevo Reglamento, el CEEUE estará compuesto por los representantes de los organismos competentes de todos los Estados miembros "y por otras partes interesadas". Los interesados participan ahora de forma directa en el CEEUE, al suprimirse el "Foro de Consulta" como órgano integrado por los interesados para cada categoría de productos que se preveía en el Reglamento anterior.

El procedimiento para la elaboración (y, en su caso, revisión) de los criterios puede iniciarse, previa consulta al CEEUE, tanto por la Comisión como por los Estados miembros, los organismos competentes y otras partes interesadas, que podrán, asimismo, asumir la dirección del proceso de elaboración (previa demostración, en el caso de otras partes interesadas, de que poseen los conocimientos necesarios y la capacidad de dirigir el proceso con neutralidad y de acuerdo con los objetivos del Reglamento). 
Para elaborar los criterios para la concesión de la etiqueta específicos a cada categoría de productos se aplican los procedimientos previstos en el Anexo I del Reglamento: (i) el procedimiento estándar, que consiste en la elaboración de un Informe técnico y de un proyecto de propuesta de criterios, que tras una fase de información pública y de reuniones con los interesados, darán lugar a un informe final y proyecto de criterios (acompañados de un manual para usuarios potenciales de la etiqueta ecológica y organismos competentes, y un manual para las autoridades encargadas de la adjudicación de contratos públicos; (ii) el procedimiento simplificado, en el que se reducen los trámites, aplicable cuando los criterios ya han sido elaborados para esa categoría de productos por otros sistemas de etiquetado ecológico EN ISO 1402 tipo I.

Una vez elaborados así los proyectos de criterios para cada categoría específica de productos, corresponde a la Comisión, previa consulta al CEEUE, realizar la propuesta definitiva de los criterios (justificando todo cambio introducido en relación con el proyecto de propuesta de criterios), así como adoptar las medidas necesarias para su establecimiento, que incluyen: (i) los “criterios de evaluación” o requisitos para evaluar la conformidad de productos específicos con los criterios de la etiqueta ecológica de la UE; (ii) la especificación de tres características medioambientales principales que pueden figurar en la etiqueta opcional con cuadro de texto; (iii) el periodo de validez de los criterios y requisitos de evaluación; (iv) el grado de variabilidad del producto permitido durante dicho periodo de validez.

La aprobación de la propuesta definitiva y de las medidas para el establecimiento de los criterios se lleva a cabo mediante el procedimiento de reglamentación con control previsto en el art. 16 del Reglamento. Según este procedimiento, la Comisión actúa asistida por un Comité compuesto por los representantes de los Estados miembros y presidido por el representante de la Comisión, que debe dictaminar favorablemente sus propuestas. Se trata de un procedimiento para la adopción de decisiones complejo, regulado por la Decisión del Consejo 1999/468/CE, por la que se establecen los procedimientos para el ejercicio de las competencias de ejecución atribuidas a la Comisión (modificada por la Decisión del Consejo de 17 de julio de 2006), en el que la Comisión comparte su competencia decisoria, además de con el Comité citado, con el Consejo y el Parlamento Europeo (que pueden oponerse a la adopción de las medidas cuando justifiquen que excede de las competencias de ejecución de la Comisión, que no 
son compatibles con el objetivo o el contenido de dicho acto; o que o no respeta los principios de subsidiariedad o de proporcionalidad).

Los criterios y las medidas necesarias para su establecimiento de cada categoría específica de productos se publicarán, una vez aprobados, en el DOUE.

En cuanto a las pautas metodológicas para la elaboración de los criterios, de acuerdo con los principios previstos en el art. 6 (que se concretan en el procedimiento de elaboración de criterios previsto en el Anexo I), éstos se determinarán científicamente teniendo en cuenta la totalidad del ciclo de vida de los productos, tomando en consideración: a) los impactos ambientales más significativos; b) la sustitución de las sustancias peligrosas por otras más seguras; c) la posibilidad de reducir el impacto ambiental por razón de la durabilidad y la reutilizabilidad de los productos; d) el equilibrio medioambiental neto entre las cargas y beneficios ecológicos, incluidos los aspectos sanitarios y de seguridad, en las distintas fases del ciclo de vida de los productos considerados; e) cuando proceda, los aspectos éticos y sociales; f) los criterios de otras etiquetas ecológicas, en especial las etiquetas medioambientales EN ISO 14024 tipo I reconocidas oficialmente, a nivel nacional o regional, cuando existan para esa categoría de productos; g) en la medida de lo posible, el objetivo de reducción de la experimentación con animales.

De esta forma, el nuevo Reglamento, al igual que los anteriores, se basa en el comportamiento ambiental del producto durante todo su ciclo de vida, lo que se conoce utilizando una expresión anglosajona como enfoque "de la cuna a la tumba" ("from cradle to grave"), que constituye una de las mayores innovaciones que introdujo, ya en el Reglamento de 1992, el sistema de etiquetado ecológico europeo respecto de los sistemas nacionales preexistentes. Con este enfoque, que ha sido incorporado después a la mayoría de los sistemas nacionales de etiquetado ecológico, se trata de identificar dónde y cuánto dañan al medio ambiente los productos en cada etapa de su ciclo de vida, empezando con la extracción de materiales (la "cuna"), siguiendo con el proceso de fabricación (incluyendo el embalaje), y acabando con el uso y valorización o eliminación final ("tumba"). La elaboración de los criterios ecológicos requiere por tanto estudios científicos sobre los impactos de los productos en cada etapa de su ciclo 
de vida y comprende el análisis de todas las interacciones del producto con el medio ambiente, incluido el uso de energía y de recursos naturales.

El nuevo Reglamento precisa, en su línea de facilitar la aplicación del sistema, que "el proyecto de propuesta de criterios se redactará de manera que sea fácilmente comprensible para los que deseen utilizarlos".

\section{La verificación de los productos y la concesión de la ecoetiqueta}

Cuando ya han sido aprobados los criterios específicos en relación a una determinada categoría de productos, para la concesión de la etiqueta a los productos individuales que la soliciten se sigue un procedimiento de verificación dirigido a "certificar que un producto cumple los criterios indicados en la etiqueta ecológica de la UE". En esta fase el protagonismo corresponde a los "organismos competentes" designados por los Estados miembros según lo previsto en el art. 4 y en el Anexo V del Reglamento ${ }^{4}$.

El organismo o los organismos competentes designados en cada Estado son los encargados de recibir las solicitudes para la obtención de la etiqueta que puede presentar cualquier operador que desee utilizarla, acompañada de la documentación pertinente, y de evaluarla para comprobar si cumple los criterios fijados para esa categoría de productos. Los operadores soportarán los costes de las pruebas y la evaluación de conformidad con los criterios de la etiqueta ecológica de la UE.

Una vez comprobado que el producto cumple con los criterios establecidos, el organismo competente asignará un número de registro al producto y lo notificará a la Comisión, que establecerá un registro común disponible en la web de la etiqueta ecológica de la UE: www.ec.europa.eu/environment/ecolabel. A continuación, el organismo competente celebrará un contrato con el solicitante sobre las condiciones de su utilización (en el Anexo IV del Reglamento se incluye un contrato tipo, a fin de las condiciones de utilización de la etiqueta sean uniformes en toda la Comunidad).

\footnotetext{
${ }^{4}$ En España, de acuerdo con lo dispuesto en el Real Decreto 598/1994, corresponde a las comunidades autónomas la designación de estos organismos competentes, siempre que cuenten en su territorio "con los medios personales y materiales con los que poder ejercer los cometidos propios de su actividad", y sin perjuicio de que la Administración General del Estado pueda designar subsidiariamente un organismo competente para aquellos casos en que no lo haya hecho la Comunidad Autónoma. En la actualidad, el organismo competente con carácter subsidiario a nivel nacional para la concesión de la ecoetiqueta europea es la Asociación Española de Normalización y Certificación (AENOR).
} 
El nuevo Reglamento, al igual que el anterior, no regula el procedimiento aplicable en el caso de denegación de la etiqueta, que hay que entender por tanto que se remite a la normativa nacional ${ }^{5}$.

Toda solicitud de concesión de etiqueta ecológica está sujeta al pago de cánones por la tramitación de la solicitud, pudiéndose establecer asimismo por el organismo competente un canon anual. El canon de tramitación es similar al preexistente pero el canon anual se contempla como de aplicación voluntaria y se reduce su importe (que ya no se calcula como un tanto por ciento sobre el volumen de ventas sino como una cantidad fija de hasta 1500 euros, con reducciones para las PYMES y microempresas, en vez de calcularse como un tanto por ciento sobre el volumen de ventas como antes).

Una vez firmado el contrato, la etiqueta podrá utilizarse en todos los países de la Unión Europea para la publicidad comercial del producto específico para el que se haya concedido. El Reglamento prohíbe expresamente "toda publicidad falsa o engañosa, así como la utilización de cualquier etiqueta o logotipo que pueda confundirse con la etiqueta ecológica comunitaria" (art. 10.1).

Compete a los organismos competentes comprobar de manera periódica que los productos a los que se ha concedido la etiqueta ecológica siguen cumpliendo los criterios y requisitos de evaluación, para lo cual se establece que el usuario de la etiqueta "permitirá que el organismo competente lleve a cabo todas las investigaciones oportunas", y, a petición del organismo competente, "permitirá el acceso a las instalaciones en las que se fabrique el producto considerado". Si un organismo competente comprueba, tras haber dado al usuario de la etiqueta la posibilidad de presentar observaciones, que un producto que lleva el logotipo de la UE no cumple los criterios correspondientes o no se utiliza conforme a las prescripciones del Reglamento, prohibirá la utilización de la etiqueta ecológica de la UE en dicho producto e informará a la Comisión de tal prohibición (art. 10).

5 En nuestro país, por aplicación de las normas generales que rigen la actuación administrativa, la denegación de su concesión habrá de ser motivada en cuanto acto limitativo de intereses legítimos (art. 54 de la Ley 30/1992, de Régimen Jurídico de las Administraciones Públicas y del Procedimiento Administrativo Común), y, en el caso de que se deniegue la etiqueta, por imperativo del art. $24 \mathrm{CE}$, esa decisión podrá ser objeto de recurso. 
El Reglamento dispone, por último, que "los Estados miembros establecerán las sanciones que deberán imponerse en caso de infracción de las infracciones del presente Reglamento y adoptarán todas las medidas necesarias para garantizar su cumplimiento" (art. 17). Por primera vez la regulación europea de la ecoetiqueta prevé expresamente la aplicación de la potestad sancionadora para garantizar su cumplimiento.

\section{MEDIDAS PARA PROMOVER LA ETIQUETA ECOLÓGICA DE LA UE}

El nuevo Reglamento pretende, como se ha expuesto, otorgar un fuerte impulso a la etiqueta ecológica de la UE y, para ello, además de simplificar y agilizar los procedimientos y de abaratar su coste, adopta las siguientes medidas:

- Se introducen mecanismos para paliar los problemas que se derivan de la coexistencia del sistema de ecoetiqueta comunitario con los de los Estados miembros ${ }^{6}$, como son: la aplicación de un procedimiento simplificado para establecer los criterios de un producto cuando se trate de criterios establecidos ya en otra ecoetiqueta (con lo que se promueve la aplicación del sistema a productos que ya cuentan con etiquetas nacionales), y la previsión, dirigida a atajar la proliferación de ecoetiquetas, de que "cuando se hayan publicado criterios de la etiqueta ecológica de la UE para una categoría de productos dada, otros sistemas de etiquetado ecológico EN ISO 14024 tipo I reconocidos oficialmente a escala nacional o regional que no abarquen esa categoría de productos en el momento de la publicación sólo podrán incluirla si los criterios elaborados con arreglo a esos sistemas son, como mínimo, tan estrictos como los de la etiqueta ecológica de la $U E$ ”. En relación a la etiqueta ecológica se dispone, de modo más flexible, que "los criterios de la etiqueta ecológica de la UE también tendrán en cuenta los criterios

\footnotetext{
${ }^{6}$ Incluso en España, aunque el etiquetado ecológico se introdujo de la mano del Derecho comunitario en 1992, pues no existían entonces etiquetas nacionales, en la actualidad coexisten con la ecoetiqueta europea diversas marcas de conformidad ecológicas. Así, por un lado, AENOR ha ido creando diversas "marcas y certificados verdes" (entre ellas, a nuestros efectos, destaca la etiqueta para asegurar la "calidad verde" de los productos y de los servicios, como es la "Marca AENOR-Medio Ambiente de producto o servicio certificado"), y, por otro lado, existen varias comunidades autónomas que han creado ya, o previsto la creación, de sus propias etiquetas ecológicas para los productos fabricados o comercializados en su ámbito regional.
} 
existentes fijados en los sistemas de etiquetado ecológico oficialmente reconocidos en los Estados miembros" (art.11).

- Se establece que los Estados miembros y la Comisión acordarán "un plan de acción específico para promover la utilización de la etiqueta ecológica de la UE" dirigido a la realización de campañas de difusión y al fomento del sistema.

- Un instrumento de incentivo de la etiqueta ecológica que introduce el nuevo Reglamento y que puede resultar muy eficaz es su vinculación con la contratación pública. La incorporación de la variable ambiental a la contratación pública se ha llevado a cabo por la Directiva 2004/18/CE, del Parlamento Europeo y del Consejo, sobre coordinación de los procedimientos de adjudicación de los contratos públicos de obras, de suministros y de servicios, que fija las condiciones en las que los poderes adjudicadores podrán introducir requerimientos de carácter ambiental y social en las licitaciones públicas ${ }^{7}$. En aquellos supuestos en los que los criterios ecológicos se toman en consideración en las fases de preparación o de adjudicación del contrato, la etiqueta ecológica de la UE constituye un elemento objetivo de valoración cuya utilidad se va a ver potenciada con la previsión por el nuevo Reglamento de que los criterios para cada categoría de productos deberán ir acompañados de un "manual para orientar a las autoridades encargadas de la adjudicación de contratos públicos sobre el uso de los criterios de la etiqueta ecológica de la UE” (Anexo I, parte A, punto 5). El Reglamento dispone, además, que los Estados deberán fomentar el uso de este Manual y, a tal efecto, considerarán la posibilidad de "fijar objetivos para la adquisición de productos que cumplan con los criterios especificados en el Manual" (art. 12.3).

No parece demasiado aventurado predecir que, con esta nueva regulación de la etiqueta ecológica de la UE que facilita y promueve su aplicación, y con la creciente preocupación ambiental de los ciudadanos, la "Flor Europea" va a convertirse por fin en un futuro próximo, en uno de los instrumentos más eficaces para incentivar la elección informada por el consumidor de los productos y servicios más respetuosos con el medio

\footnotetext{
7 Sobre la valoración del factor ambiental en la contratación pública, véase nuestra obra Derecho Ambiental Administrativo, Dykinson, Madrid, 10ª edición, pp. 442 ss.
} 
ambiente, en el marco de la política de producción y consumo sostenibles de la Unión Europea ${ }^{8}$.

\footnotetext{
${ }^{8}$ Véase la "Comunicación de la Comisión al Consejo y al Parlamento Europeo de 18 de junio de 2003. Política de productos integrada. Desarrollo del concepto del ciclo de vida medioambiental" [COM (2003) 302 final]. El objetivo de esta política es reducir los impactos ambientales originados por los productos durante todo su ciclo de vida, centrándose en las tres etapas del proceso de decisión que condicionan el impacto ambiental del ciclo de vida de los productos: la aplicación del principio "quien contamina paga" a la hora de fijar su precio, su diseño ecológico, y la elección informada del consumidor, aspecto éste último en el que la etiqueta ecológica juega un papel central.
} 